\title{
RoboCupJunior: Learning with Educational Robotics
}

\author{
Elizabeth Sklar ${ }^{1}$, Amy Eguchi², and Jeffrey Johnson ${ }^{3}$ \\ 1 Dept. of Computer Science, Columbia University \\ 1214 Amsterdam Ave \\ New York, NY 10027, USA \\ sklar@cs.columbia.edu \\ 2 School of Education, University of Cambridge \\ 17 Trumpington Street \\ Cambridge, CB2 1QA, UK \\ eae24@hermes.cam.ac.uk \\ 3 Dept. of Design and Innovation \\ The Open University \\ Milton Keynes, MK17 8QH, UK \\ j.h.johnson@open.ac.uk
}

\begin{abstract}
The RoboCupJunior division of RoboCup is now entering its third year of international participation and is growing rapidly in size and popularity. This paper first outlines the history of the Junior league, since it was demonstrated in Paris at RoboCup 1998, and describes how it has evolved into the international sensation it is today. While the popularity of the event is self-evident, we are working to identify and quantify the educational benefits of the initiative. The remainder of the paper focuses on describing our efforts to encapsulate these qualities, highlighting results from a pilot study conducted at RoboCupJunior 2000 and presenting new data from a subsequent study of RoboCupJunior 2001.
\end{abstract}

\section{Introduction}

In 1998, Lund and Pagliarini demonstrated the idea of a children's league for RoboCup, using robots constructed and programmed with the LEGO Mindstorms kit to play soccer [8]. Since then, RoboCupJunior has evolved into an international event where teams of young students build robots to compete in one of three challenges: soccer, rescue and dance 6 9,18]. While the atmosphere at these events is electric, from an intellectual standpoint one asks: "what are the students learning from these activities?"

It would be too easy to say that because the students are interacting with technology they are learning something worthwhile. However, this appeared to be the conventional wisdom as children began using computers in the early days — from the 1970's into the 1990's. Today researchers are questioning this stance. For example, Healy suggests that no more than $10 \%$ of the available 
software for children has any educational value [5]. Reeves reminds us that "fifty years of media and technology comparison studies have indicated no significant differences in most instances" [17]. And Snyder warns that we are becoming "blinded by science" [19.

In our work, we are questioning the same "obvious" relationship between robotics and educational outcomes. Rather than focus just on the technology itself, we are examining the overall learning environment that results when groups of students participate in team robotic activities. We begin by outlining the history of RoboCupJunior, illustrating its growing popularity on an international scale. Second, we discuss results of a pilot study conducted at the RoboCupJunior tournament held in Melbourne in 2000 [18]. Based on this study, we make several observations about factors that we believe contributed to the success of the event. Third, we present results from a subsequent study conducted at RoboCupJunior 2001 held in Seattle. Finally, a comparison of the two studies is made, some conclusions are drawn and future directions are described.

\section{History}

RoboCupJunior began as a research project in Lund's LEGO lab at the University of Aarhus in Denmark. The idea was to explore the use of the LEGO Mindstorms platform for robotic soccer. The initial growth of the initiative in 1999 and early 2000, exclusively at Lund's lab, expanded the breadth of the project and increased the number of different activities offered. The focus was on continued exploration of the educational possibilities of robotics, particularly in terms of teaching young students about Artificial Life.

\subsection{First Steps: 2000}

At RoboCup Euro 2000 in Amsterdam, Kröse and others used the Interactive LEGO Football (ILF) software and robot design developed by Lund and Pagliarini to hold a one-day RoboCupJunior workshop [6]. Fifty children, ages 13-16, from 8 schools formed 12 teams to play one-on-one soccer. In the morning, each team was given a robot and taught how to use the ILF software. In the afternoon, there was a tournament with prizes given out at the end of the day.

Simultaneously during 2000, preparation was getting underway in Melbourne, Australia, in advance of the RoboCup 2000 conference. A committee comprised of local school teachers and representatives from universities and industry developed a blueprint for RoboCupJunior competitions involving a curriculum based, student driven approach. Three challenges were devised, each requiring a different level of sophistication.

- A dance challenge was designed for primary school children (up to age 12). Students would build robots that would move to music for up to two minutes. Creativity was emphasized. From a technical standpoint, this was presented as an entry-level event, since it was possible to participate using 
simple robots that only employ motors and no sensors. The event itself was exciting and innovative. Some children even dressed in costume themselves and performed alongside their robots (see figure 17a).

- A sumo challenge was designed for middle school children (ages 12-15). Two robots followed wiggly black lines and competed for possession of a central circular region on the playing field. This was presented as a middle-level event; only one robot was needed for each team and the environment was essentially static. The only dynamic elements were the two robots; they had limited interaction and did not need to respond to each other, only to changes in their own location on the playing field. (see figure 15).

- A soccer challenge, inspired by Lund's project and videotapes of the senior RoboCup leagues, was designed by and for secondary school students (ages $12-18$ ). Two teams of two robots each played on a special field, $150 \mathrm{~cm} \times$ $75 \mathrm{~cm}$ in size. The floor of the field used a greyscale mat and the ball was an electronic device that emitted infra-red (IR) light 9. The rules of play were developed from the RoboCup F-180 League rules (see figure 10).

\subsection{The Next Phase: 2001}

During 2001, several regional events were organized around the world. In April, a workshop was offered at CHI-Camp, an activity-centered child-care program

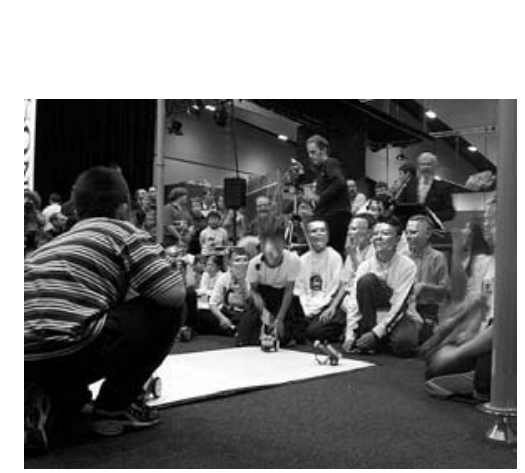

(a) dance

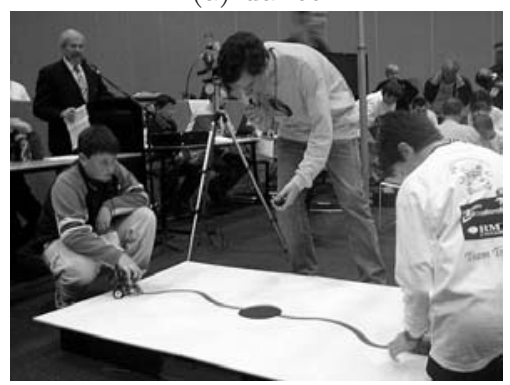

(c) sumo

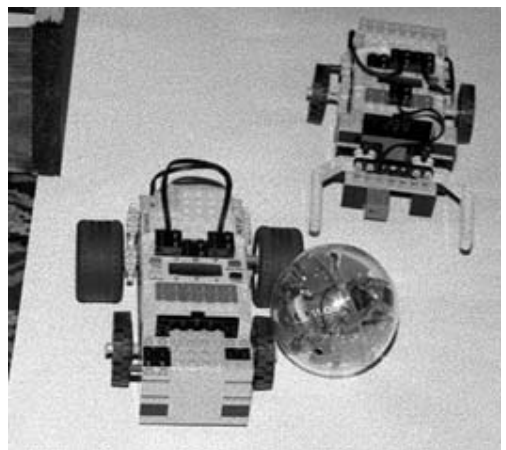

(b) soccer

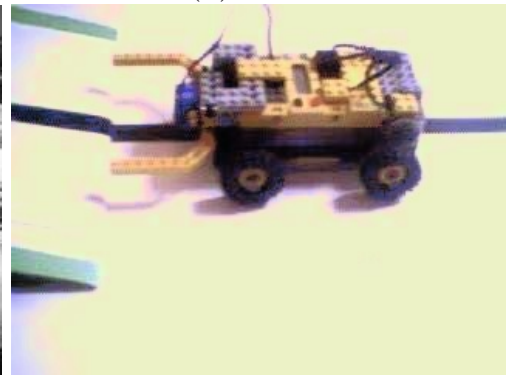

(d) rescue

Fig. 1. RoboCupJunior challenges. 
for children of attendees to the annual CHI (Computer Human Interaction) conference, held in Seattle. In June, a mini RoboCupJunior tournament was held at the Autonomous Agents conference in Montréal. Six teams entered the one-day tournament, from four middle and high schools, involving six teachers, three parents and 53 students. Also in June, a RoboCupJunior tournament was incorporated into the German Open in Paderborn.

At the Japan Open 2001, Nomura and others organized a RoboCupJunior event in which 84 children participated. Most were from Fukuoka City; one team came from Tokyo and ten students came from Busan, Korea. About half of the participants were elementary school students (ages 9-12) and the other half were junior high school students (ages 13-15). Six activities were defined within several courses, and participants chose a course based on their interest and experience levels. Separate workshops focused on robot assembly and programming. Only soccer was offered (two-on-two). Some of the rules were outside the bounds of the official RoboCupJunior rules. For example, one track allowed remote controlled robots. The autonomous track used a visible light ball and a special field designed and produced by EK Japan. This field used beacons to indicate the positions of the goals and to help the robot locate itself within the field, instead of the greyscale floor mat used on the fields at RoboCupJunior 2000.

In August 2001, the second international RoboCupJunior tournament was held in Seattle at RoboCup 2001. Twenty-five teams from four countries participated: Australia, Germany, the UK and the USA. There were 104 participants (students and mentors). Again, three challenges were offered. The most significant differences from RoboCupJunior 2000 were that there were no age restrictions on challenges, tertiary (undergraduate) teams were permitted and the sumo/line-following challenge was replaced by a rescue event:

- A rescue challenge was designed as middle-level event. This was a timed event where one robot competed at a time. The background of the field was white, and the robot was required to follow a black line through a simulated disaster scenario. This was presented as a middle-level challenge. Again, only one robot was needed for each team. There were no dynamic elements, but accurate control of the robot based on light sensor readings is essential and surprisingly difficult. As well, the uneven terrain with a change in pitch where the robot reached a ramp and a bump in the path between the flat field and the ramp made the course even more challenging (see figure $1 \mathrm{~d}$ ).

The rules for soccer and dance were similar to RoboCupJunior 2000. The soccer field was enlarged to $122 \mathrm{~cm}$ by $183 \mathrm{~cm}$, a factor of nearly double. This was done to accommodate increased maximum size for the robots, to allow kits other than the LEGO Mindstorms; for example, the FischerTechnik Mobile robot, where the basic building block that houses the microprocessor is considerably larger than the equivalent LEGO component.

In September 2001, the Australian National RoboCupJunior championship was held in Melbourne in association with Interact 2001 [21. During the months preceding the tournament, five states conducted eliminations. In all, over 200 
teams and 500 students participated. The finals were conducted in an auditorium with hundreds of spectators joining in to create an exciting atmosphere. This event received acclaim from industry representatives and VIP guests, to the extent that organizers were officially congratulated during a sitting of Victorian State Parliament.

\section{Studies}

\subsection{Related Work}

The notion of using robotics as a tool for education is relatively new. The recent availability of off-the-shelf robotic kits such as LEGO Mindstorms, FischerTechnik Mobile Robot and Elekit SoccerRobo have made it technically feasible and affordable for secondary and even primary schools to integrate robotics in the classroom. Most studies of this phenomenon focus on curricular ideas - explanations of what subject areas could be enhanced through robotics and methodologies for how to use the robots 10111 .

Other work has focused on outcomes. One such study was conducted at a large public university in Indiana where most of the students work full-time while trying to complete a technical degree [16. Students take an introductory technology, engineering and computer science course in their first year (ETCS 101). A pilot version of the course, revolving around a team-based robot project using LEGO kits, was first offered in Fall 1999 and introduced into the regular curriculum in Fall 2000. The course focuses on teamwork as well as technical aspects. Students are assigned specific roles within their teams, such as material specialist and webmaster. The primary objective is to improve retention of engineering students in later subjects and ultimately to graduation. Statistical results show that the goal has been met thus far. Students who took the introductory course prior to inclusion of the robotics project had a $37 \%$ chance of leaving the ETCS program, while after inclusion of the robotics project, there is only a $5 \%$ likelihood of students leaving the program.

A comprehensive study was conducted by Wagner 24], examining the effects of robotics in primary classrooms (ages 8-12). The overriding question in her study was to determine if use of robotics in science curriculum had a greater effect on children's performance on science achievement tests than use of battery powered (non-robotic) manipulatives. The study found that use of robotics did not significantly improve general science achievement, though the use of robotics did improve programming problem solving (though not general problem solving). The study also highlighted that there are many mitigating factors when conducting a study of this sort. For example, students participating in the study are aware that they are subjects and may act differently if they were not being observed. Wagner also notes that the study opened up more questions than it answered.

Verner recognized the educational value inherent in the senior leagues of RoboCup 22, noting that RoboCup embodies project-based education, a pedagogy currently very popular in engineering curricula. He identified various components of project-based learning, e.g., motivation, problem solving, teamwork 
and cooperation, and administered a questionnaire to participants at RoboCup 1997 focusing on these aspects. He reported statistics on composition of team membership, length of participation in RoboCup, motivational factors and curricular subjects (for the upcoming RoboCup summer school). The study revealed that the most important motivational factor for all participants was "to apply and test [their] ideas". The study also revealed that a low motivational factor was "prospective career opportunities". The most popular curricular summer school subject was a simulator building workshop.

In the next year, Verner performed a follow-up study [23], using similar methodologies, but performing a more comprehensive analysis of the results. He broke down statistics according to role in order to analyze differences between professors and students. Motivational aspects were examined as well as applicability of general science and engineering subjects such as computer vision and multi-agent collaboration. The curricular statistics are not surprising, for example $100 \%$ of simulation league team members say that they are concerned with multi-agent collaboration, while none of them are concerned with control circuits. The motivational results reveal that professors view RoboCup as slightly more motivating than students in terms of attitude towards the subject matter and practical issues, while students view the fun of the robot soccer game as more motivating than professors.

In Verner's results, it is interesting to note the contrast between intrinsic and extrinsic motivational aspects. These factors have been studied by psychologists in basic terms such as rewarding birds for pressing levers, and more recently in relation to education [7]. Intrinsic rewards are those felt internally by a person achieving a task, simply out of satisfaction for having performed the task. Extrinsic rewards are those offered by outsiders, either verbally or materially (e.g., trophies), often having no obvious relation to the task being performed. In general, it has been found that extrinsic rewards can harm a person's natural intrinsic motivation to perform a task if the reward is given at one time and then taken away. For example, children who like to draw in their free time and are subsequently rewarded for drawing are afterward less likely to draw in their free time when the reward is no longer offered.

Verner's results show that the intrinsic rewards offered for participation in RoboCup are far more significant than the extrinsic rewards. Namely intrinsic factors: "a positive attitude towards the subject, the method and the framework for research and education suggested by the program" and "opportunity to apply your ideas and reinforce practical and teaching/learning skills" were considered highly motivating by $71 \%$ of respondants. Whereas the extrinsic factor: "ambition to cope with the RoboCup challenges and win a reward at this prestigious professional contest and forum" was considered highly motivating by only $35 \%$ of respondants.

\subsection{The RoboCupJunior Challenge}

The work presented here attempts to bring these various issues to bear within the RoboCupJunior arena. Two pilot studies were conducted, in 2000 and 2001, 
and the results of both are presented in this section. While the methodology for collecting data is similar to those above (i.e., in the form of surveys and questionnaires), the challenge of extracting valid and useful results is difficult for two reasons. First, the subjects are children who may not be able to measure accurately various motivational factors on a 5-point scale (e.g.). Second, the international composition of RoboCupJunior brings forth language and cultural issues more than in the senior leagues, since young students are less likely to be able to factor out cultural issues than adults.

\subsection{Pilot Study: RoboCupJunior 2000}

RoboCupJunior 2000 involved about 40 teams of children, 8 to 19 years old; one team was from the USA, one was from Germany and the remaining 38 teams were from Australia. We conducted interviews with twelve of the teachers who entered teams in the tournament, with the general stated goal of investigating the educational value of RoboCupJunior.

The interviews were conducted in two parts. First, background information was gathered on paper. Then interviews were video-recorded for subsequent analysis. The background questions were used to establish the experience level of the teacher and the specifics of the school environment, e.g., age groups, genders and subjects taught. The interview questions were divided into two parts. The first section focused on how each teacher used RoboCupJunior in their curriculum. The second section concentrated on comparing RoboCupJunior with other types of projects and activities, like science fairs, school plays, math team, etc. The questions asked about the influence of RoboCupJunior on students' behavior and motivation, with respect to schoolwork and teamwork.

The selection of the teachers to be interviewed was quite opportunistic. During the two days of the tournament, we approached teachers when they were not busy with their teams and asked them to be interviewed. The interviews took about forty minutes each. Our subjects may not represent all the teachers at the competition fully. However, it is worth noting that teachers from 9 out of the 13 schools whose teams participating in the soccer challenge were interviewed.

The analysis is based on notes taken during the interviews as well as transcripts of the videotapes. We acknowledge that the sample size is small and that the sample is biased - the respondents were teachers who believed in the initiative because they chose to enter in the first place. Additionally, we feel that the group sampled is technically more advanced than the average population of all teachers — most teach science and/or technology curriculum; several have degrees in engineering.

Our pilot study revealed remarkable consensus of opinion amongst the teachers. RoboCupJunior fits in with existing robotics curriculum; is highly motivating for participants; advances both academic and personal development skills; teaches teamwork and tolerance of others; and may attract girls into robotics as well as boys. If these attributes generalize to other teachers in other school systems, it could be seen that RoboCupJunior is a very positive educational initiative. We note that in subsequent investigations in countries other than Aus- 
tralia, we have found the inclusion of robotics in school curricula is unusual, as school systems vary greatly from one nation to another and even from one region to another within a single nation, depending on the government organization of school systems within a given country.

In addition, we relate several observations in terms of motivation, transfer and gender issues. All of the teachers reported that the RoboCupJunior competition itself was a motivating factor, particularly because: it is an international event, it imposes an absolute deadline (i.e., the date of the conference is fixed) and it gives children an entry-level role in the complex and stimulating field of robotics research. Several teachers commented that the context of RoboCupJunior the fact that the young entrants participated alongside the senior competitors, some of the top robotic scientists and engineers in world — was a tremendous motivating factor for them and their students.

About half of the teachers thought the children who participated behaved better during their preparation for the competition than they did during other classroom activities. Most teachers thought RoboCupJunior was helpful in other areas of their students' schooling, although some related concern expressed by a few parents and other teachers about time taken away from other lessons in order to prepare for RoboCupJunior.

Less than ten percent of the participating children were girls, but most teachers surmised that expansion of the dance challenge may encourage girls in future (the dance challenge was limited to age 12 at RoboCupJunior 2000). In Melbourne, most of the participants were boys; indeed, less than ten percent of participants were girls. No girls attended the robot soccer or sumo tournament, though a few did help teams prepare at school but were unable to attend the tournament. Girls did participate in the dance challenge, which was restricted to primary age children (12 years and under). This increased participation could either be due to the creative nature of the dance challenge, the younger age group, or both.

\subsection{Second Study: RoboCupJunior 2001}

At RoboCupJunior 2001, we surveyed not only mentors, but also the students. We present the results of both surveys in this section, starting with the mentors. All participants were requested to participate in the paper-and-pencil survey. In addition, students participated in a 20-30 minute videotaped interview. The results presented here are based on the paper surveys and some notes from the videotaped interviews.

Mentors. Out of 25 mentors, 16 completed the survey (64\%). Among the mentors, there are seven classroom teachers, eight parents (including one classroom teacher and two club leaders), one camp counselor, and one former RoboCupJunior student. Six of the teachers work at the middle and high school level; one is from a university. Their students ranged in age from 11 to 24 . 
Although most of the mentors' teaching experiences are focused in technical areas (math, physics and technology), teachers from other areas were also involved. These included biology, social studies and theology.

Among the teachers who responded to the survey, one teacher was from the US, two teachers were from Australia and four were from Germany. Only two teachers responded that they have robotics activities included in their curriculum - one from Germany and one from Australia. Both teach in public (government funded) schools. Overall, 62.5\% of the mentors indicated that their students attend public schools, while $12.5 \%$ attend private (students/families pay fees for them to attend) 1 .

Our research is centered around trying to identify what the students are learning from their RoboCupJunior activities. We asked both mentors and students to consider 13 specific skills and indicate whether they felt their involvement in RoboCupJunior had helped or hurt each of these skills, or if there was no effect. The selection of the specific skills listed was based on transcripts of the video interviews conducted at RoboCupJunior 2000 [18].
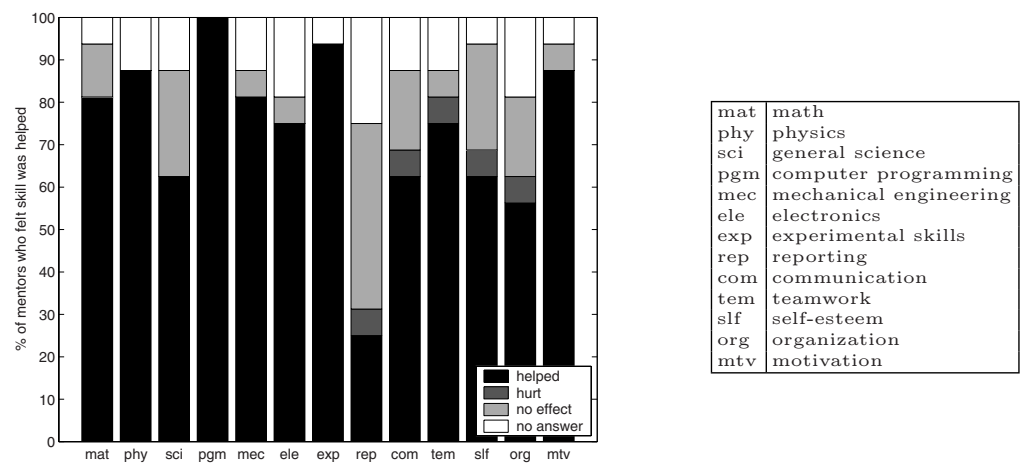

Fig. 2. Effects on various skills, according to mentors (2001).

The results are shown in figure 2, The bars illustrate the number of mentors who indicated whether each skill was helped, hurt, etc. For example, $80 \%$ of the mentors indicated that they thought their students' math skills were helped through their preparation for RoboCupJunior; approximately $12 \%$ of the mentors indicated that they thought that the RoboCupJunior preparation had no effect on their students' math skills; and $8 \%$ of mentors did not respond to the question. The overall consensus is that all the skills named were helped more than they were hurt; indeed those who felt that all skills were either helped or not affected far outnumbered those who indicated that any given skill was hurt.

One notable exception is in regard to reporting skills. Mentors ranked reporting skills as being helped less than other skills (students also ranked reporting skills low, as described in the section below). This could be due to the lack

${ }^{1} 25 \%$ of the mentors did not respond to the question of whether their students attend public or private school. 
of activities such as keeping journals and writing lab reports, because perhaps mentors might not expect that work in robotics can help such skills - however, these types of activities are extremely important in scientific research, since researchers must be able to communicate their discoveries. Teams were encouraged to give presentations at a RoboCupJunior workshop, after the tournament was over, describing their preparation. All of the teams were invited to submit papers to the informal Junior workshop, but none of them did. Further emphasis on reporting as part of the tournament itself will help promote development of this skill set.

Students. Out of 82 students, 43 completed the surveys (52\%). First, we focus on the demographic information, examining the number of students who participated in the event and the number of students who completed the survey, grouped according to country. Table 1 shows breakdowns both on an individual basis, as well as by team. These figures are relevant as the subsequent data is presented on an individual basis or team-wise, depending on which reporting mechanism is appropriate given the data being presented. In either case, it may be helpful to refer back to these tables.

Table 1. Participation, by country (2001).

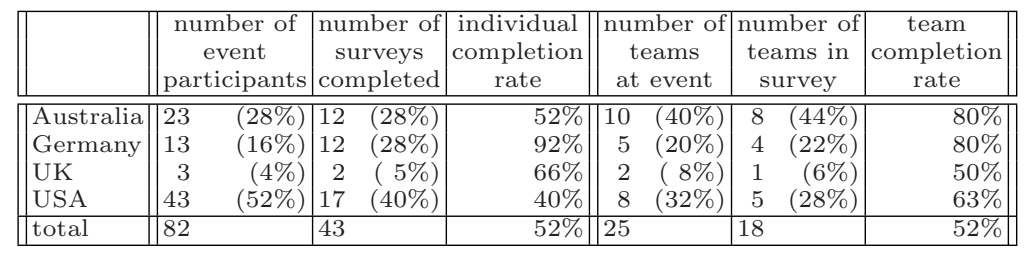

The gender gap was narrowed in 2001. Overall, $11 \%$ of participants were female. The German teams were all-male, but the other nations had mixed teams. The Americans had more females altogether; there were three all-female teams (one American, two Australian).

Next, we look at the age distribution, as shown in figure 3. Most participants are in high school, between the ages of 16 and 18.

Several questions in the survey focused on the teams' preparation, asking what was the span of preparation time, how frequently the teams met and how long each meeting lasted. Figure 4 shows these data graphically. Each mark on the plot represents one of the teams who completed the survey. The position of each mark indicates the average number of hours of preparation made by each team, per week. There are no patterns here, except to note that $77 \%$ of the teams spent at least 2 hours per week preparing. Some teams spent very little time, preparing everything at the last minute. Other teams spent up to a year preparing, meeting regularly after or during school.

We also asked questions about the type of adult supervision and help received by the teams while they prepared (figures 2 (a) and 2 (b)). Although these 


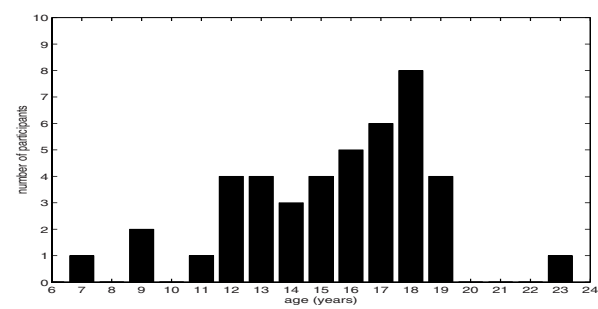

Fig. 3. Distribution of ages amongst participants (all participants, 2001).

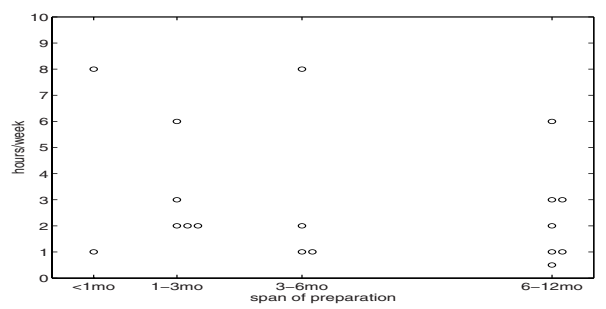

Fig. 4. Comparison of preparation times (2001).

Table 2. Adult supervision and help (2001).

\begin{tabular}{|c|c|}
\hline $\begin{array}{c}\text { number of } \\
\text { adults helping }\end{array}$ & teams \\
\hline 0 & $3(17 \%)$ \\
\hline 1 & $8(44 \%)$ \\
\hline 2 & $2(11 \%)$ \\
\hline 3 & $1(6 \%)$ \\
\hline 4 & $2(11 \%)$ \\
\hline 5 & $2(11 \%)$ \\
\hline
\end{tabular}

(a)

\begin{tabular}{|l|r|}
\hline level of adult assistance & teams \\
\hline \hline almost none & $6(33 \%)$ \\
a lot in the beginning & $4(22 \%)$ \\
a lot throughout & 1 \\
mixed & $7(6 \%)$ \\
\hline
\end{tabular}

(b)

questions were asked on an individual basis, the figure contains an average for each team, since it is more appropriate to analyze these data on a per team basis. For the most part, there was consensus amongst team members. A few teams had wide discrepancy, but that is likely due to the fact that on many teams, members joined at different times, so those who were participants early on might have received more help from mentors than those who joined after the team had been working for a while. Some students commented that they (the mentors) were only needed to give the students the keys to the closet where the robots were stored or to go out for pizza. Others, particularly the younger students, said that they received "a lot of help throughout" their preparation.

At RoboCupJunior 2000, all teams used the LEGO Mindstorms platform. Some teams used LEGO without modifying it in any way, others made considerable mechanical and electronic developments of their own, including spinning mechanisms for kicking and non-standard sensors. In 2001, other platforms were used, namely the Fischer-Technik mobile robot (16\% of teams) and the Tetrixx 
kit (4\% of teams) developed at Gerhard Kraetzschmar's lab at the University of Ülm [4]. The LEGO Mindstorms kit was used by $80 \%$ of the teams.

All of the teams that did not use LEGO were German teams. One of the German teams that participated in RoboCupJunior 2000 using LEGO, progressed to the Tetrixx platform for 2001. Typically, the choice of which robot kit to use depends on the local culture and which platform is most familiar to the students and/or mentor. For example, at the Japan Open in 2001, almost all the teams used the Elekit Soccer Robot kit produced by EK Japan; only one team used LEGO Mindstorms. Since RoboCupJunior 2002 is being held in Japan, it is expected that there will be more diversity in platforms including LEGO, Fischer-Technik, Tetrixx and Elekit.

The heart of the survey asked the students to consider whether any of a list of skills had been affected by their participation in RoboCupJunior. They were asked to rank 13 skills as having been either helped, hurt or not affected by their participation. The answers, based on individuals' data, are shown in figure 5 Most students felt that their programming, mechanical and electronics skills had been improved.
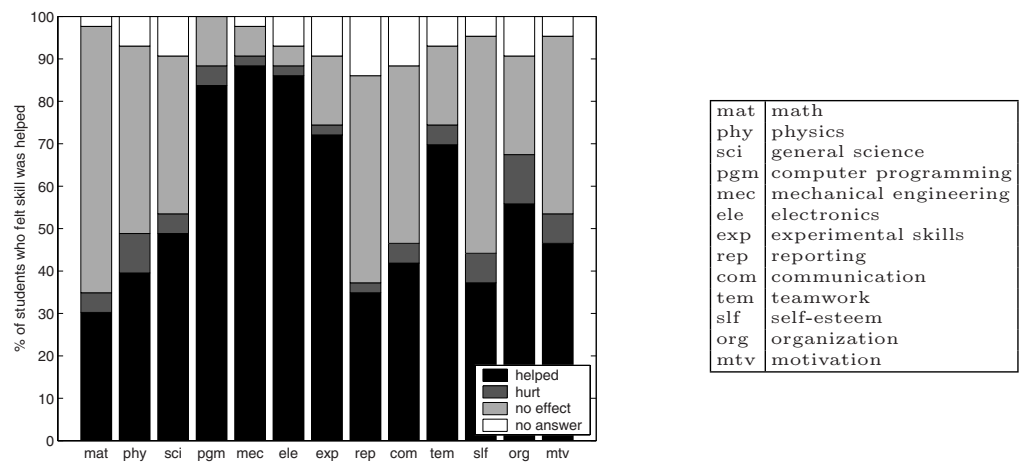

Fig. 5. Effects on various skills, according to students (2001).

\section{Analysis}

Methodology. It is interesting to contrast the results of the studies from both years (2000 and 2001), although a statistical comparison cannot be made since the survey methodology differed from one year to the next. The 2000 study used open-ended questions. Subjects were prompted only when necessary (quite infrequently). The 2001 study built upon the previous year's results, consisting of a combination of open-ended and closed-ended (i.e., multiple choice) questions. The problem with open-ended questions is that the results are often difficult to tally, particularly to calculate any statistical significance due to slight variations in responses. The problem with closed-ended questions is that the respondents are often influenced by the answers already supplied (i.e., the multiple choices) 
and rarely provide in-depth information about their experiences or thoughts. Our compromise was to design the closed-ended questions based on data collected in 2000, while also including some open-ended questions in order to highlight differences between the two years' events. Future studies will build on results from both years.

Mentors. One large difference between RoboCupJunior 2000 and RoboCupJunior 2001 was in the number of classroom teachers who acted as mentors. In 2000, almost all of the mentors were teachers; in 2001, a smaller fraction of them were teachers. This is due to several factors. First, the timing of the event (August) was such that schools were closed, and teachers and students were involved with holidays, summer camp, etc. Still, most teams (91\%) were organized through some type of institution — school, summer camp or community group. This statistic is similar to that of 2000, though in that year a larger percentage of mentors were classroom teachers and had robotics included in their curriculum.

The second factor is a political issue. A recent trend in US education is toward standardized testing. This is the case in Washington state, as well as California, Massachusetts, New York and many other states. Students are tested several times during their K-12 years, as a basis for matriculation and graduation. Teachers are forced to narrow their curriculum and "teach to the test", as the performance of individual schools are being tracked closely. This limits the amount of curricular freedom the teachers have, hence more creative programs such as robotics are typically eliminated.

Age of Students. In 2000, the dance challenge was limited to primary grades (ages 12 and under), and several teams were class efforts involving upwards of 20 students per team. In 2001, only $12 \%$ of the students were in this age group. This might be because about half the students were from overseas, and it is difficult for younger students to travel abroad to participate in the event. Or, it could be because there is still a myth among educators and parents that robotics is too difficult for young children. We should encourage younger generation and promote robotics activities since such children have more potential to develop various skills through robotics activities.

Skills. It is especially interesting to compare the 2001 mentors and students ratings for how the various skills were effected (see figures 2 and 5). Overall, more of the mentors consider that RoboCupJunior has positive effects than the students, as highlighted by figure 6. It could be considered that it is more difficult for students to assess the effects objectively than it is for mentors. Moreover it is harder for students to assess abstract skills, such as communication, selfesteem and organization, than it is for them to evaluate concrete skills, such as mathematics, physics and programming. In future studies, we will re-examine the survey to come up with more effective ways of asking students about abstract skills. 

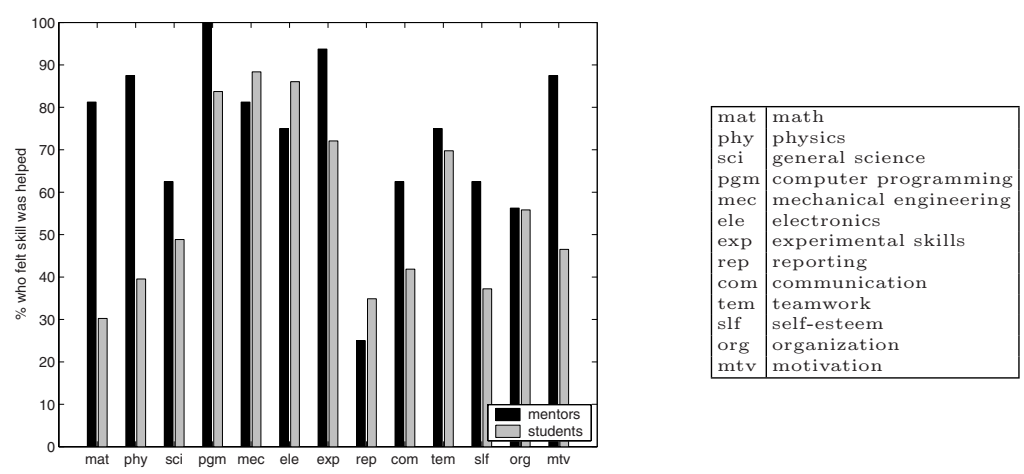

Fig. 6. Variation in skill ratings between mentors and students (2001).

Future Participation. About $70 \%$ of both students (65\%) and mentors $(75 \%)$ expressed their willingness to participate in the RoboCupJunior international event again next year. Moreover, $75 \%$ of mentors are willing to participate in RoboCupJunior locally and nationally. This suggests that RoboCupJunior is so appealing and challenging that the participants become enthusiastic enough to continue working on it. This also shows that RoboCupJunior has great potential to grow to be an effective educational activity to enhance students learning in various ways. In order to make this happen, our future work involves continued promotion of RoboCupJunior activities, development of effective curriculum and clear assessment the effectiveness of RoboCupJunior activities in order to provide better educational environments for participants.

\section{$5 \quad$ Future Directions}

The motivational aspects of computer games, which have great attraction for children [20], are also found in robotic soccer. Among the 25 teams who participated in 2001, most of them spent more than one hour, and three fifths of the teams spent more than two hours, for one preparation meeting. This suggests that robotics activities are challenging and attractive enough to make students focus on their work for long periods of time. It also implies that, in order to merge this activity into regular curricula, teachers need to make effective plans to adjust the length of an activity into the regular class period without distracting students' motivation or to extend the class period to give their students enough time to explore ideas.

The emphasis on teamwork in RoboCupJunior allows students with a variety of interests and abilities an opportunity to pick their own challenges while contributing to the progress of the whole, an experience which nurtures the varied and multiple intelligences of each participant [2]. One way of applying this philosophy would be for teams in the same location to use a variety of platforms. Note that to date, all teams which have more than one robot have used the same platform for all their robots. This is not a requirement, so it will be interesting 
to see if this changes in the future as the teams' approaches become more sophisticated and advanced; or as teachers seek ways to challenge teams comprised of members with varying levels of experience with robotics and RoboCupJunior.

The expense to obtain new and varied platforms may hinder the accumulation of a diverse inventory. For the promotion of RoboCupJunior activities, it is important that we continue to include and highlight various platforms so that students and mentors are able to learn about the options that exist and are able to select those that are best for the team. Demonstrations at RoboCupJunior events can help make students and mentors aware of the variety of platforms available on the market today.

Although the results presented here are preliminary, the work fulfills a need in the community to examine the effects of these types of projects, to find standard and effective ways of evaluating them, and to define curricula that fosters and takes advantage of the positive elements identified. RoboCupJunior is an on-going, "plan-do-see" research program that provides effective educational activities to children of all ages, all around the world.

\section{Acknowledgements}

We are ever grateful to Brian Thomas for his tireless efforts behind the scenes of RoboCupJunior, in Australia and internationally. We thank all the students, mentors and organizers who have given of themselves and their time to prepare for and participate in RoboCupJunior events around the world.

\section{References}

1. Coradeschi S. and Malec J., How to make a challenging AI course enjoyable using the RoboCup soccer simulation system. In RoboCup-98: Robot Soccer World Cup II, Lecture Notes in Artificial Intelligence (LNAI) vol. 1604, Springer Verlag, 1998.

2. Gardner, H., Frames of Mind: The Theory of Multiple Intelligences, 1983.

3. Gonzalez, A., Digital divide closes - but schools aren't ready, USA Today, April 26, 2000 .

4. http://www.tetrixx.de

5. Healy, J., Failure to connect: how computers affect our children's minds, New York: Simon \& Schuster, 1998.

6. Kröse, B., Bogged, R., and Hietbrink, N., Programming robots is fun: RoboCup Jr. 2000. In Proceedings of Belgium-Netherlands AI Conference 2000, 2000.

7. Lepper, M. and Henderlong, J., Turning "play" into "work" and "work" into "play": 25 years of research on intrinsic versus extrinsic motivation. In Intrinsic and extrinsic motivation: The search for optimal motivation and performance, Academic Press, 2000.

8. Lund, H.H. and Pagliarini, L., Robot Soccer with LEGO Mindstorms. In RoboCup98: Robot Soccer World Cup II, Lecture Notes in Artificial Intelligence (LNAI) vol. 1604, Springer Verlag, 1998.

9. Lund, H.H. and Pagliarini, L., RoboCup Jr. with LEGO Mindstorms. In Proceedings of ICRA2000, New Jersey: IEEE Press, 2000. 
10. Martin, F.G., Ideal and Real Systems - A Study of Notions of Control in Undergraduates Who Design Robots. In Constructionism in Practice: Designing, Thinking, and Learning in a Digital World, Y. Kafai and M. Resnick (eds.), 1996.

11. Miglino, O., Lund, H.H. and Cardaci, M., Robotics as an Educational Tool. Journal of Interactive Learning Research, 10:1, 1999.

12. Nomura, T., personal communication (email), December 17, 2001.

13. Papert, S., Mindstorms: Children, Computers and Powerful Ideas, New York: BasicBooks, 1980.

14. Papert, S., The Children's Machine, New York: BasicBooks. 1991.

15. Piaget, J. To Understand Is To Invent, New York: The Viking Press, Inc., 1972.

16. Pomalaza-Ráez, C. and Groff, B.H. Retention 101: Where Robots Go... Students Follow, In Proceedings of the 2002 American Society for Engineering Education Annual Conference and Exposition, 2002.

17. Reeves, T. A Research Agenda for Interactive Learning in the New Millenium. In Proceedings of the World Conference on Educational Multimedia, Hypermedia \& Telecommunications (EdMedia99), 1999.

18. Sklar, E.I., Johnson, J.H. and Lund, H.H. Children Learning from Team Robotics: RoboCup Junior 2000 Educational Research Report, Technical Report, The Open University, Milton Keynes, UK, 2000.

19. Snyder, T., Blinded By Science, The Executive Educator, 1994.

20. Soloway, E., How the Nintendo Generation Learns, Communications of the ACM, 34(9), 1991.

21. Thomas, B., personal communication (email), January 8, 2002.

22. Verner, I.M. The Value of Project-Based Education in Robotics. In RoboCup-97: Robot Soccer World Cup I, Lecture Notes in Artificial Intelligence (LNAI) vol. 1395, Springer Verlag, 1997.

23. Verner, I.M. The Survey of RoboCup '98: Who, How and Why. In RoboCup-98: Robot Soccer World Cup II, Lecture Notes in Artificial Intelligence (LNAI) vol. 1604, Springer Verlag, 1998.

24. Wagner, S.P. Robotics and Children: Science Achievement and Problem Solving. Journal of Computing in Childhood Education, 9:2, 1999. 